\title{
Una defensa de la justificación etnocéntrica*
}

\author{
GLENDA SATNE \\ Departamento de Filosofía \\ Universidad de Buenos Aires \\ glesatne@mail.abaconet.com.ar
}

\begin{abstract}
Resumen: En este artículo me propongo analizar los argumentos que presenta Kalpokas para rechazar la concepción rortiana de la justificación y sostener, por oposición a ésta, la independencia lógica de la justificación y de la reforma respecto al consenso comunitario. Argumento que la posición sostenida por Kalpokas resulta incoherente; en particular se lleva mal con una posición pragmatista consecuente, tanto en lo que se refiere a la historicidad de los criterios de justificación, como en cuanto a la posibilidad de prescindir de un punto de vista espacial y temporalmente determinado. Sostengo, asimismo, que una concepción etnocéntrica de la justificación puede incluir una elucidación racional del cambio y reforma de nuestros criterios epistémicos, con lo cual se desarticula la acusación de arbitrariedad que Kalpokas atribuye a la visión rortiana de los mismos.
\end{abstract}

Palabras clave: justificación, etnocentrismo, consenso global

Daniel Kalpokas defiende ${ }^{1}$ la idea según la cual es imprescindible distinguir el concepto de justificación del concepto de justificación etnocéntrico, es decir, aquel que es relativo a una comunidad, en un tiempo y un lugar determinados. Esto es preciso, según su perspectiva, porque es necesario diferenciar lo que es correcto de lo que nos parece correcto, ya que de otro modo nuestra práctica justificatoria colapsaría en meras afirmaciones de hecho y no alcanzaríamos conocimiento. Sólo de esta manera la justificación sería lógicamente independiente de lo que opina la mayoría. Para apoyar esta idea, retoma algunas tesis de Putnam, ${ }^{2}$ en particular aquellas formuladas en los cinco principios epistémicos. Su estrategia consiste en defender la adecuación de estas tesis frente a algunas reformulaciones críticas que éstas han sufrido en particular por parte de Rorty. ${ }^{3}$

El objetivo principal de mi artículo es mostrar que ninguno de los argumentos presentados por Kalpokas con el propósito de señalar la invia-

* Durante la realización de este trabajo conté con una beca de la Fundación Antorchas (Argentina).

${ }^{1}$ Todas las referencias a D. Kalpokas son de su artículo "El debate Putnam-Rorty sobre la naturaleza de la justificación" que aparece en este número, pp. 119-126.

${ }^{2}$ H. Putnam, Realism with a Human Face, Harvard University Press, Cambridge, 1990, p. 22 .

${ }^{3}$ R. Rorty, "Putnam and the Relativist Menace", The Journal of Philosophy, vol. XC, no. 9, 1993, p. 449. 
bilidad de la propuesta rortiana resultan adecuados, en particular, que el mejor modo de entender el criterio de aceptabilidad racional idealizada de Putnam es entenderlo como aceptabilidad para nosotros en nuestra mejor versión. Además, argumentaré que este criterio es el más adecuado para dar cuenta tanto de la naturaleza de la justificación como de las reformas en nuestros estándares de epistémicos.

Kalpokas presenta los cinco principios de la justificación de Putnam y en seguida el rechazo de Rorty de los dos primeros. Respecto del primero (objetividad), Rorty lo reformula ${ }^{4}$ diciendo que es una cuestión de hecho si un enunciado $p$ está justificado, ya que es una cuestión sociológica. Según Kalpokas, esto equivale a abandonar "aquella dimensión desde la cual se evalúan las razones que se ofrecen en justificación del enunciado en cuestión. Esto es, la consideración epistémica de por qué se acepta a $p$ como verdadero es relegada por la observación de cómo considera la comunidad a p" (Kalpokas, p. 120). Por otra parte, Penelas ${ }^{5}$ interpreta que aquí el problema no es que la justificación sea una cuestión sociológica, sino la compatibilidad entre esta afirmación rortiana y el rechazo del segundo principio (independencia con respecto a la opinión de la mayoría). Propone una reformulación del mismo en términos de independencia respecto de la totalidad de la comunidad, para mostrar que la mayoría puede estar equivocada aun si la justificación es una cuestión de hecho. Concuerdo en este punto con Penelas; en efecto, en la perspectiva rortiana, la independencia de la justificación respecto del consenso sólo es concebible en la medida en que se toma en cuenta a la totalidad de la comunidad. De este modo, no hay una instancia lógicamente independiente de dicho consenso global que a su vez eventualmente permita considerar equivocada a esta totalidad, ya que sólo a través de la experiencia futura, con la constitución de un nuevo punto de vista en la comunidad, podrá evaluarse que los enunciados anteriormente justificados ya no lo están. ¿̇Implica esto que el planteo rortiano es básicamente sociológico y no puede concebirse en términos normativos? En mi opinión, Kalpokas y Penelas confieren un estatuto equivocado a la afirmación de Rorty de que la justificación es una cuestión sociológica. Esta afirmación debe entenderse a la luz de una distinción entre dos planos distintos: por un lado, es una afirmación que hace el teórico, el sociólogo o el filósofo; es decir, la justificación es una cuestión de hecho para aquel que, ubicado desde el punto de vista del observador, describe la práctica justificatoria. Por otro lado, ubicándose en el interior del juego de dar y

${ }^{4}$ R. Rorty, ibid., p. 449.

${ }^{5}$ F. Penelas, "La justificación como hecho social”, pp. 127-134, en este número. 
pedir razones, a través del cual se desarrolla la práctica, la afirmación de que un enunciado está justificado resulta ser una cuestión de derecho. Al no hacer esta distinción, ambos hacen colapsar los dos aspectos, con las siguientes consecuencias: el primero debe negar la importancia, para la teoría de la justificación, del consenso que existe en una comunidad respecto de determinados enunciados. Al resaltar únicamente el aspecto normativo de la cuestión, infiere errónamente que el concepto de justificación debe trascender el contexto en el cual se produce. Al mismo tiempo juzga mal las virtudes de la teoría rortiana de la justificación para dar cuenta de que ésta se produce sobre la base de buenas razones, en particular basándose en aquellas que resultan buenas razones para nosotros. Por su parte, Penelas, al concebir la cuestión de la justificación como un mero hecho sociológico, privilegia ilegítimamente el punto de vista del observador de la práctica, dejando inexplicado cómo es que la justificación es una cuestión de derecho. Presumo que es por ello que desconfía de la probidad de la teoría rortiana para dar cuenta de la reforma de nuestros estándares de justificación y de la evaluación de los mismos. El punto crucial es que Rorty nos dice que lo que constituye que la justificación sea una cuestión de hecho es "el que seamos capaces de calcular si $S$ estaba en buenas condiciones, dados sus propios intereses y valores y los de sus iguales, de afirmar $p$ ". ${ }^{6}$ Penelas parece omitir que esas "buenas condiciones" suponen la dimensión normativa, aun si no se trata de considerar las razones sub specie aeternitatis; es decir, independientemente de nuestras capacidades de justificar $p$ ante quienes nos rodean. El problema de Penelas es que destaca la función del observador de la práctica eliminando la reflexión acerca de las "buenas condiciones" que nos llevan a justificar nuestros enunciados. Por el contrario, debería resaltar, como lo hace Dewey, ${ }^{7}$ no el modo contemplativo, sino el modo operativo en el que los procesos de conocimiento se llevan a cabo.

\section{III}

Para defender la plausibilidad del principio de la independencia, la idea de que la justificación es independiente de lo que opine la mayoría, es necesario, según Kalpokas, suponer que hay alguna instancia independiente de la misma. Esta instancia, sugiere Putnam, está constituida por aquello que aceptaríamos en condiciones ideales de investigación. ${ }^{8}$ Ahora bien, ¿cómo

${ }^{6}$ R. Rorty, ibid., p. 450.

7 J. Dewey, Reconstruction in Philosophy (1920), en Jo Ann Boyston (comp.), Works, Southern Illinois University Press, Carbondale (III), 1988 [versión en castellano: La reconstrucción de la filosofía, Aguilar, Buenos Aires, 1955]. Según el uso habitual, en adelante citaré esta edición de las Obras completas con las siglas EW (Early Works), MW (Middle Works) o LW (Later Works).

${ }^{8}$ Para Kalpokas las condiciones epistémicas ideales determinan que "toda vez que tenemos por justificada una creencia asumimos que tales condiciones han sido logradas" y nos sirven, 
podemos entender esta apelación a una aceptabilidad ideal? Asumiendo los compromisos pragmatistas que tanto Rorty como Putnam están dispuestos a mantener, no es posible concebir dicho punto de vista como el punto de vista neutral, o punto de vista de Dios; el privilegio del punto de vista del agente nos impide el compromiso con dicha posibilidad de "ver las cosas desde ninguna parte". 9 Es por esta razón que Rorty, al interpretar esta propuesta, nos dice: "No puedo ver qué puede significar aceptabilidad racional idealizada sino aceptabilidad para nosotros en nuestra mejor versión."10

Contra la identificación entre estos dos conceptos, Kalpokas da dos argumentos distintos:

El primero intenta mostrar que el candidato rortiano no es plausible, ya que supone una identificación entre lo que es mejor y lo que nos parece mejor que no permite distinguir entre mejores y peores normas de justificación, razón por la cual no puede dar cuenta de las reformas de nuestros estándares epistémicos. La noción de arreglárnoslas mejor no sería equivalente a la de parecer que nos las arreglamos mejor, aquélla señala la existencia de mejores y peores estándares, esta última meras preferencias arbitrarias. De este modo, lo que Kalpokas intenta mostrar es que el concepto de reforma conlleva la idea según la cual el que un cambio sea bueno o malo es lógicamente independiente de que nos parezca bueno o malo. Por este motivo, concluye que no podemos dejar de aceptar la independencia lógica tanto de la reforma como de la justificación respecto de la opinión de la mayoría de nuestros pares.

Una primera respuesta a esta objeción es que, al igual que en el caso de Rorty, tanto desde el punto de vista de Kalpokas como del propio Putnam, no es posible acceder a lo que es mejor si no es desde nuestro punto de vista. Adviértase que ninguno de los dos tendrían que estar en condiciones de adoptar un punto de vista trascendente a la comunidad en la cual se logra el consenso necesario respecto de cuáles son las creencias que resultan justificadas. Si esto es así, ambos deberían aceptar que esa distinción es interna a cada uno de los marcos conceptuales y entonces lo que es mejor no puede determinarse independientemente de lo que nos parece mejor.

Por otra parte, no es cierto que la diferencia que agrega nuestro punto de vista en una concepción de lo mejor sea la del juicio individual y arbitrario.

a su vez, de criterio normativo para criticar nuestras actuales prácticas toda vez que dichas condiciones no se cumplen (p. 123).

${ }^{9}$ El rechazo de una concepción tal es reconocido por Putnam: "Creo que el núcleo del pragmatismo - el de James y Dewey, si no el de Peirce- fue la insistencia en la supremacía del punto de vista del agente. Si encontramos que no tenemos más remedio que adoptar cierto punto de vista, usar determinado 'sistema conceptual', cuando estamos inmersos en una actividad práctica, entonces no debemos hacer al mismo tiempo la afirmación de que ése no es verdaderamente el modo en que las cosas son en sí mismas" (H. Putnam, Las mil caras del realismo, Paidós, Barcelona, 2000, p. 86).

${ }^{10}$ R. Rorty, op. cit., p. 451. 
No es cierto que cualquier cosa pueda llegar a parecernos mejor. Y ésta es la idea que está detrás de la objeción mencionada. Kalpokas parece considerar que el concepto de parecernos mejor es un concepto puramente valorativo, entendiendo los valores como algo meramente arbitrario. Sin embargo, lo que llega a parecernos mejor puede ser visto, tal como sugieren las reflexiones de Dewey, como el producto racional de nuestra interacción con el medio. ${ }^{11}$

Kalpokas nos pide un método tal que su bondad "no depende de lo que yo crea o me parezca sino de los resultados efectivos que produce". Ahora bien, el núcleo del pragmatismo consiste justamente en no distinguir entre "lo que me parece mejor" y "los resultados efectivos que algo produce". Lo que nos parece mejor no se traduce en términos de deseos impulsivos o puramente subjetivos, sino que resulta valorado o ponderado aquello que conduce a los mejores resultados, en un sentido de éxito práctico. ${ }^{12}$ Dentro de nuestro marco conceptual podemos trazar la distinción que nos muestra, sin adoptar un punto de vista trascendente, que hay algo que determina lo que es mejor en el interior de nuestra práctica. Así, no cualquier cosa puede resultarnos útil [convenient], con lo cual no es correcta la objeción de Kalpokas según la cual cualquier cambio en nuestros estándares de justificación es aceptable. De este modo, me parece que el recurso a la utilidad es suficiente para revisar sin arbitrariedad, a la luz de nuestra experiencia, los criterios a través de los cuales mantenemos nuestra práctica.

El segundo argumento está destinado a mostrar que el concepto rortiano de aceptabilidad para nosotros en nuestra mejor versión, o es inadecuado a la hora de dar cuenta de la justificación y de la consideración de nuestros estándares de justificación como mejores y peores; o bien es ambiguo porque entraña elementos inconciliables para satisfacer intereses teóricos contrapuestos. El problema es el lugar que los otros ocupan en relación con un nosotros determinado. Según Kalpokas, por un lado, el contenido de nuestro paradigma de corrección representado por nosotros en nuestra mejor versión debe abarcar lo suficiente como para posibilitar la inclusión de los otros pasados y los otros futuros, y excluir esquemas conceptuales inconmensurables; pero, por otro lado, no debe incluir al otro en un sentido universal y debe afirmar el nosotros. De tal supuesta incoherencia extrae la necesidad de postular como único criterio de corrección posible aquel que se comprometa con algún tipo de justificación independiente respecto de lo que esté justificado contextualmente; en este sentido, tal criterio debe ser universal: debe ser común a nosotros y a ellos.

Como hemos argumentado anteriormente, Rorty no postula que dicha dimensión universal sea necesaria ni tampoco posible. No es difícil notar

${ }^{11}$ J. Dewey, The Quest for Certainty, 1929, LW, 4.207 [versión en castellano: La busca de la certeza, México, Fondo de Cultura Económica, 1952].

12 Dewey da cuenta de este aspecto en J. Dewey, op. cit, pp. 207-208. 
cuán cerca está este argumento de Kalpokas de la defensa de un punto de vista en todo independiente de alguna comunidad determinada. En segundo lugar, tiene como corolario indeseado la desestimación del carácter histórico de los estándares epistémicos y la relatividad a intereses y valores de los mismos, que Putnam había enunciado como imprescindibles a toda concepción pragmatista. En este punto, quizás sin notarlo, Kalpokas ha ido más lejos de lo que él mismo se había permitido avanzar. Tal como se lo señala Penelas, ${ }^{13}$ la defensa de una independencia en sentido fuerte, expresada en el segundo principio, parece conducir a quien quiera comprometerse con algún tipo de pragmatismo a sostener una posición incoherente. O bien aceptamos los principios de historicidad y relatividad a intereses y concebimos la justificación como históricamente condicionada, con lo cual no podemos sostener que hay un sentido de estar justificado que no depende del punto de vista de un agente determinado; o bien renunciamos a la concepción pragmática del lenguaje y concebimos que términos como “justificación" significan algo que está más allá de las prácticas internas a la comunidad. Conservar ambas opciones implica sostener al mismo tiempo dos cosas incompatibles.

Esto es, para ser coherente con los principios según los cuales nuestros estándares de justificación resultan ser productos históricos, y reflejan nuestros intereses y valores, es necesario afirmar el nosotros como sujeto relevante en la justificación por oposición a un sujeto universal.

Nuevamente considero que, al contrario de la opinión de Kalpokas y Penelas, esta aceptación del nosotros no produce un conflicto respecto a la evaluación de nuestros criterios de justificación. Conceder ese lugar privilegiado a nuestro propio punto de vista no invalida que en el seno de la comunidad tengan lugar tensiones producidas por la confrontación entre sujetos con intereses contrapuestos o por la confrontación entre dicha comunidad y otras comunidades, de modo tal que la aceptación del etnocentricismo no requiere negar la confrontación con puntos de vista contrapuestos, aun si negamos, para ser coherentemente pragmatistas, la idea según la cual hay estándares válidos para toda comunidad.

\section{IV}

Otra objeción al punto de vista etnocéntrico como criterio de evaluación de nuestros estándares es que no permitiría fundamentar nuestros cambios de vocabulario y criterios en razones. Pero si esto es así, concluye Kalpokas, entonces no tiene sentido hablar de criterios o estándares mejores y peores; ni tampoco de progreso.

${ }^{13}$ F. Penelas, op. cit. 
Por el contrario, creo que podemos concebir este cambio como racionalmente motivado en el marco rortiano. Considero que el consecuencialismo deweyeano, como sucedía en el caso de la distinción entre lo que es mejor y lo que nos parece mejor, nos permite contestar a esta objeción. La consecución de nuestros fines y el logro de nuestros deseos aparecen como motivadores racionales de los cambios. A través de la apelación rortiana al "continuum medios-fines" de Dewey: "la idea según la cual uno cambia de deseos a medida que descubre qué sucede cuando trata de conseguir lo que una vez deseó", ${ }^{14}$ es posible explicar las reformas de nuestros sistemas conceptuales como racionalmente motivadas.

Esta propuesta implica modificar nuestra concepción de lo que constituye una buena razón. Dicho cambio se sostiene en una crítica al modo en que el criterio presentado por Kalpokas funcionaría para presentar razones que justifiquen un cambio de un vocabulario por otro. El problema es que es difícil discernir cuál sería el contenido concreto de dicho criterio de "condiciones epistémicas ideales" en cada caso. Al modo del imperativo categórico kantiano, este criterio parece ser más bien abstracto y su aplicación concreta depende de la interpretación que cada comunidad particular le dé en su propio seno. De este modo, o bien se torna irreconocible como criterio; o bien resulta tan históricamente condicionado como la propuesta rortiana, y pierde su universalidad.

En el primer apartado mencioné que considero que es posible pensar la justificación etnocéntrica desde el plano normativo y no meramente desde el punto de vista descriptivo. Por un lado, la justificación, tal como lo propone Penelas en su texto, puede describirse como un "hecho social". Por otro lado, sin embargo, es necesario dar cuenta de cómo los cambios en nuestros estándares de justificación se producen sobre la base de razones y son evaluables en términos de mejores y peores. Mi propuesta es, al contrario de la presentada por Kalpokas, subrayar el cambio de método que el pragmatismo propone. Éste nos incita a abandonar las concepciones tradicionales acerca de la razón y de la relación de las representaciones con la experiencia. No se trata, como propone Kalpokas, de encontrar razones trascendentes a las prácticas o comunes - universal y trascendentementea las mismas; al contrario, se trata de modificar nuestra concepción de la racionalidad con base en la consideración de la práctica. La propuesta coherentemente pragmatista para evaluar racionalmente cambios en los criterios de justificación es la siguiente:

Un cambio significativo que resultaría de llevar el método experimental desde la física al hombre se refiere al importe de las normas, principios, reglas. Al hacer esta transferencia, éstos, junto con las doctrinas y credo en torno a lo bueno y los bienes, se reconocerían como hipótesis. En vez de estar rígidamente

${ }^{14}$ Rorty apela a este concepto deweyneano en R. Rorty, ibid., p. 80 (nota 39). 
fijados, se los trataría como instrumentos intelectuales que hay que poner a prueba y confirmar -y alterar-a través de las consecuencias producidas al actuar según ellos. ${ }^{15}$

En suma, he argumentado que no se entiende en qué sentido Kalpokas puede hablar de lo que es mejor sin hacer referencia a lo que nos parece mejor. Considero que en dicha estrategia idealizadora reaparece aquel intento infructuoso del realista de ver el mundo desde ninguna parte. Asimismo, he argumentado que hay una tensión entre la tentación universalista de Kalpokas y los propios principios epistemológicos putnamianos que establecen el carácter histórico y la relatividad a intereses y valores de nuestras normas y estándares de justificación. Por otra parte, he cuestionado el olvido de la dimensión normativa en el que Penelas incurre al subrayar únicamente el punto de vista del observador respecto de la justificación. Además, en discordancia con ambos, considero que a la luz de las observaciones que hemos hecho anteriormente, resulta plausible afirmar que en un marco teórico etnocéntrico es posible dar cuenta del cambio, de las reformas, y de la evaluación de los criterios de justificación en términos de mejor y peor, y concebir dichos cambios como racionalmente motivados. He intentado mostrar que en el interior de nuestra práctica hay algo que determina lo que es mejor. Las reformas de los criterios y estándares tienen su origen en nuestra percepción de la diferencia entre lo que es conveniente en nuestra práctica y lo que nos parece conveniente en principio. También con este recurso es posible dar cuenta de los cambios en los criterios epistémicos como racionalmente motivados: no cualquier cambio es aceptable.

Recibido el 19 de junio de 2002; aceptado el 26 de noviembre de 2002

${ }^{15}$ J. Dewey, op. cit., LW, 4221. Las cursivas son mías. 